\title{
Analysis of AT hydaulic system based on the logical-shift formula
}

\author{
Jiangjiang Li ${ }^{1, a}$, Jinliang $X^{2} e^{2, b}$ \\ ${ }^{1}$ Tianjin Vocational Institute,Tianjin,China \\ ${ }^{2}$ Langfang Polytechnic Institute, Hebei,China \\ a18622205953@163.com, b305546638@qq.com
}

Key words: Logical analysis, Logical-shift formula,Hydraulic system design ,Solenoid valve status

Abstract. Based on the shift actuator table of AT and the Principle of the hydraulic system ,it is easy to get the logical-shift formula with the help of the logical analysis, which could help to design AT hydraulic system .Besides, it conducts a feasibility analysis of their shift solenoid valve control state .

\section{Introduction}

There are three main design principles to restricate the direction of hydraulic oil in the design of hydraulic control system[1][3].

Principle 1: the designed hydraulic system was capable to complete the required basic shifting according to the actuator components table.

Principle 2: Due to the characteristics of mechanical structures, we have to consider some requirements in the design of hydraulic system that some of actuator components must interlock to prevent the interference between some actuator components; Besides, we also have to prevent the interference between the gear, mainly to prevent the case of jumping gear that the shift gear should happen in the adjacent gear and should not occur between the interval gear.

Principle 3: When the shifting solenoid valve failed, the hydraulic system should be able to achieve some gear to ensure the car to run for some time which called fail-safe capability

The relationship among the principles mentioned above was that the principle2 was meaningful on the basis of the principle1; the principle 3 was basic on the principle1, the principle 2 could be considered the modifications, complement and complete of the hydraulic system.

\section{Design of the hydraulic shift system}

Shift-logic formula.Based on the analysis above , we can get two sets of hydraulic logic diagram with using the logical analysis : 

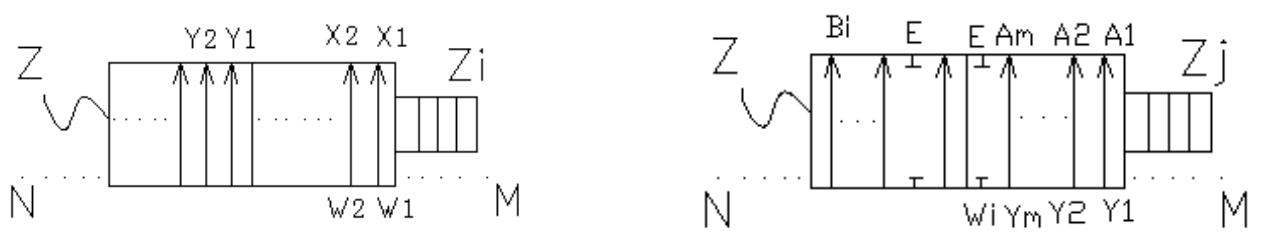

$$
\begin{array}{ll}
W_{1}=\left(\overline{Z_{1}} Z\right) X_{1} \overline{N_{1}}+\left(Z_{1}+\bar{Z}\right) Y_{1} \overline{M_{1}} & Y_{1}=\left(\overline{Z_{2}} Z\right) A_{1} \bar{N}+\left(Z_{2}+\bar{Z}\right)\left(A_{1} \bar{M}+E\right) \\
W_{2}=\left(\overline{Z_{2}} Z\right) X_{2} \overline{N_{2}}+\left(Z_{2}+\bar{Z}\right) Y_{2} \overline{M_{2}} & Y_{2}=\left(\overline{Z_{3}} Z\right) A_{2} \bar{N}+\left(Z_{3}+\bar{Z}\right)\left(A_{2} \bar{M}+E\right) \\
W_{3}=\left(\overline{Z_{3}} Z\right) X_{3} \overline{N_{3}}+\left(Z_{3}+\bar{Z}\right) Y_{3} \overline{M_{3}}(1) & Y_{3}=\left(\overline{Z_{4}} Z\right) A_{3} \bar{N}+\left(Z_{4}+\bar{Z}\right)\left(A_{3} \bar{M}+E\right) \\
\vdots & \vdots \\
W_{S}=\left(\overline{Z_{i}} Z\right) X_{n} \overline{N_{n}}+\left(Z_{i}+\bar{Z}\right) Y_{m} \overline{M_{m}} & Y_{S}=\left(\overline{Z_{j}} Z\right) A_{n} \bar{N}+\left(Z_{j}+\bar{Z}\right)\left(A_{m} \bar{M}+E\right) \\
& W_{i}=\left(\overline{Z_{j}} Z\right) E_{n} \bar{N}+\left(Z_{j}+\bar{Z}\right) B_{m} \bar{M}
\end{array}
$$

Note: represents the control terminal of ON.i solenoid valve $\mathrm{i}=12 \ldots \mathrm{n}, \quad$ means spring control terminal; less than that is ; and represent the main oil pressure;, 、 equal to locking oil, 、 represents inversion of locking oil, means that may lead to or lead to oil spills, which is based on the action of implementation of component

We put (1) (2) as a whole and call it a hydraulic logical design unit, in fact, through the analysis of the design of automatic transmission hydraulic system ,it is cleared that the design of the hydraulic system is the combination of several such hydraulic logical design unit, and specific combination is based on transmission

\begin{tabular}{|c|c|c|c|c|c|c|c|c|c|c|}
\hline & & $\mathrm{C} 1$ & $\mathrm{C} 2$ & $\mathrm{C} 3$ & B1 & B2 & B3 & F1 & $\mathrm{F} 2$ & $\mathrm{P}$ \\
\hline & & & & & & & & & & ) \\
\hline $\mathrm{R}$ & 1 & & & 0 & 0 & 0 & & 0 & 0 & \\
\hline $\mathrm{N}$ & & & & 0 & 0 & & & & & \\
\hline & 1 & & & & & & 0 & $P$ & 0 & \\
\hline & 2 & 0 & & & & & 0 & & 0 & \\
\hline & 3 & 0 & 0 & & & & 0 & & & \\
\hline $\mathrm{D}$ & 4 & 0 & 0 & 0 & & & & & & \\
\hline & OD & & 0 & 0 & 0 & & & 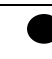 & & \\
\hline
\end{tabular}
machinery parts and actuator components table .

Design of the hydraulic shift system .Based on the actuator table of AT(AMT, see Table 1 )[4], we can use the shift-logic formula mentioned above to design hydraulic shift system and get the situation of shift solenoid valve.

Table 1 actuator table of AMT

With using the shift-logic formula (1) (2) above, we can get the hydraulic shift system diagram met actuator table of AMT mentioned above:
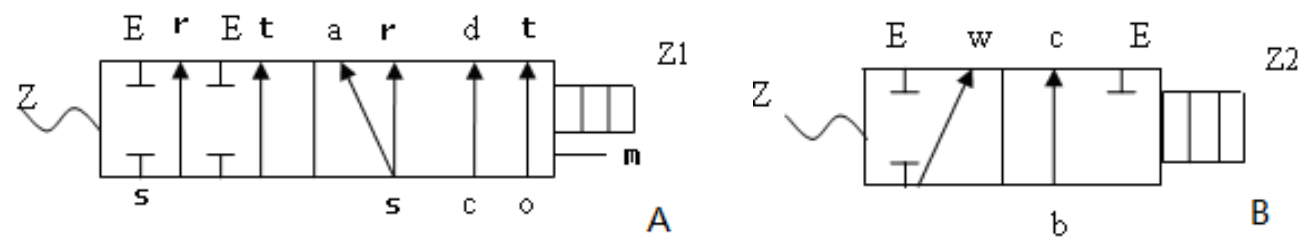

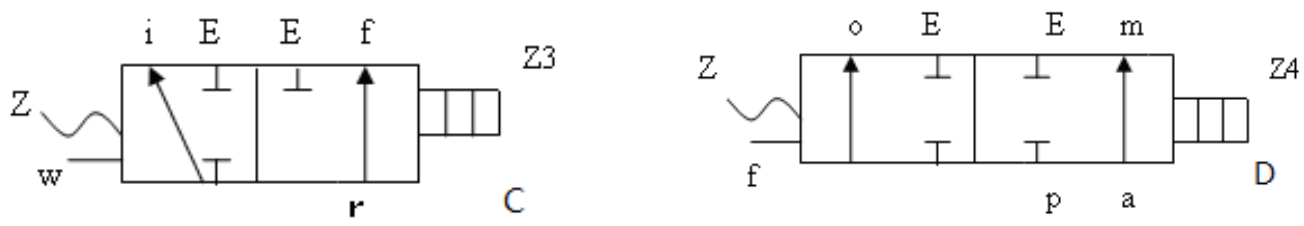

Control strategy analysis[2] of solenoid valve .There will be some analysis of control state of the solenoid valve (in accordance with the actuator table of AMT mentioned above):

P position: there are almost no oil flow in the hydraulic system under the parking gear.

$\mathrm{N}$ position (s, p have oil): $Z 1$ is powered on and $s=r, s=(\overline{Z 1}+Z) E$, $o=(\overline{Z 1}+Z) t, \quad Z 2$ is powered off and $b=(\bar{Z} Z 2) c, \quad Z 3$ is powered on and $r=(\overline{Z 3}+Z) i, \quad Z 4$ is powered on and $p=(\overline{Z 4}+Z) o, a=(\overline{Z 4}+Z) E$, so it is clear that: $s=(\overline{Z 3}+Z) i, \quad p=(\overline{Z 4}+Z)(\overline{Z 1}+Z) t$;

R position (s, p、B2 have oil): $Z 1$ is powered on and $s=r, s=(\overline{Z 1}+Z) E$, $o=(\overline{Z 1}+Z) t, \quad Z 2$ is powered off and $b=(\bar{Z} Z 2) c, Z 3$ is powered on and $r=(\overline{Z 3}+Z) i, \quad Z 4$ is powered on and $p=(\overline{Z 4}+Z) o \quad a=(\overline{Z 4}+Z) E$, so it is clear that: ${ }^{s=(\overline{Z 3}+Z) i}, \quad p=(\overline{Z 4}+Z)(\overline{Z 1}+Z) t$, another oil goes through $\mathrm{B} 2$ directly;

D position ( $\mathrm{s}, \mathrm{p} 、 \mathrm{~b}$ have oil):

1 shift: $Z 1$ is powered on and $s=r, Z 2$ is powered on and $b=(\overline{Z 2}+Z) w, Z 3$ is powered off and $r=(\bar{Z} Z 3) f$ so $s=(\overline{Z Z 3}) f, \quad Z 4$ is powered off and $p=(\bar{Z} Z 4) E, \quad a=(\bar{Z} Z 4) m$, because $Z 1$ is powered on,so a has no oil

2 shift: $Z 1$ is powered off and $s=(\bar{Z} Z 1) a, s=r, o=(\bar{Z} Z 1) t, c=(\bar{Z} Z 1) d, Z 2$ is powered on and $b=(\overline{Z 2}+Z) w, Z 3$ is powered off and $r=(\bar{Z} Z 3) f, Z 4$ is powered off and $a=(\bar{Z} Z 4) m, p=(\bar{Z} Z 4) E$, so it is clear that: $s=(\bar{Z} Z 4)(\bar{Z} Z 1) m$, $s=r=(\bar{Z} Z 3) f$

3 shift: $Z 1$ is powered off and $s=(\bar{Z} Z 1) a, s=r, o=(\bar{Z} Z 1) t, c=(\bar{Z} Z 1) d, Z 2$ is powered off and $b=(\bar{Z} Z 2) c, Z 3$ is powered off and $r=(\bar{Z} Z 3) f, \quad Z 4$ is powered off and $p=(\bar{Z} Z 4) E, \quad a=(\bar{Z} Z 4) m$, so it is clear that : $s=(\bar{Z} Z 4)(\bar{Z} Z 1) m$, $b=(\bar{Z} Z 2)(\bar{Z} Z 1) d$

4 shift: $Z 1$ is powered off and $s=(\bar{Z} Z 1) a, s=r, o=(\bar{Z} Z 1) t, \quad c=(\bar{Z} Z 1) d, Z 2$ is powered off and $b=(\bar{Z} Z 2) c, Z 3$ is powered on and $r=(\overline{Z 3}+Z) i, Z 4$ is powered off and $p=(\bar{Z} Z 4) E, \quad a=(\bar{Z} Z 4) m$, so it is clear that : $s=(\bar{Z} Z 4)(\bar{Z} Z 1) m$, $b=(\bar{Z} Z 2)(\bar{Z} Z 1) d, \quad s=r=(\overline{Z 3}+Z) i$ 
OD shift: $Z 1$ is powered off and $c=(\bar{Z} Z 1) d, s=r, o=(\bar{Z} Z 1) t, Z 2$ is powered off and $b=(\bar{Z} Z 2) c, \quad Z 3$ is powered on and $r=(\overline{Z 3}+Z) i, \quad Z 4$ is powered on and $p=(\overline{Z 4}+Z) o, \quad a=(\overline{Z 4}+Z) E$, so it is clear that : $b=(\bar{Z} Z 2)(\bar{Z} Z 1) d$, $s=(\bar{Z} Z 1)(\overline{Z 3}+Z) i, \quad p=(\bar{Z} Z 1)(\overline{Z 4}+Z) t$

Note: correspond to shift solenoid control of A, B, C, D and $===1$ when solenoid powered outage, $===0$ when solenoid powered on, $\mathrm{Z}$ represents spring control terminal, $\mathrm{Z}=0$ when solenoid powered outage valve and $\mathrm{Z}=1$ when solenoid powered on, so during solenoid powered outage and when solenoid powered on

Through the analysis above, the control state of solenoid valve can be showed in Table 2:

Table 2 control state table of solenoid valve

\begin{tabular}{|c|c|c|c|c|c|c|}
\hline & position & $\mathrm{Z} 1$ & $\mathrm{Z} 2$ & $\mathrm{Z3}$ & $\mathrm{Z4}$ & components \\
\hline \multicolumn{2}{|c|}{$\mathrm{R}$} & off & Off & on & off & C3 B1 B2 \\
\hline \multicolumn{2}{|c|}{$\mathrm{N}$} & on & Off & on & on & C3 B1 \\
\hline \multirow{5}{*}{$\mathrm{D}$} & 1 & on & On & off & off & B3 \\
\hline & 2 & off & On & off & off & C1 B3 \\
\hline & 3 & off & Off & off & off & $\mathrm{C} 1 \mathrm{C} 2 \mathrm{~B} 3$ \\
\hline & 4 & off & Off & on & off & $\mathrm{C} 1 \mathrm{C} 2 \mathrm{C} 3$ \\
\hline & OD & off & Off & on & on & $\mathrm{C} 2 \mathrm{C} 3 \mathrm{~B} 1$ \\
\hline
\end{tabular}

The control state of the solenoid valve had been designed by the use of logical analysis, where the basic state of the solenoid valve are normally open.

\section{Summary}

It can be seen from the analysis above that the use of logical analysis to design and analysis of the hydraulic shift system has a good effect, the shift-logic formula mentioned in the text has some universal in the design of shift system.

\section{References}

[1] Huang Zongyi. Modern Theory and Design of Automatic Transmission [M]. Shanghai: Tongji University Press, 2008,9. (chinese)

[2] Li JiangJiang, You Mingfu. Hydraulic system design of the automatic transmission Based on the logical analysis [J]. Machine Tool \& Hydraulics, 2011.(chinese)

[3] Zongyi Huang. The principle and design of AT used on modern car, edited by Tongji University Publications, Shanghai, china (2009), p.61-116. (In Chinese)

[4] Guoqi He, Fangming Tan, Shaoliang Wang etc. Mechanical transmission. Vol.30 (2009), p.69-73. (In Chinese)

[5] Liming Cao, Qinwu Geng etc. The maintenance essence of auto automatic transmission, edited by Mechanical industry Publications, (2010), p.448-450. (In Chinese) 